\title{
Disturbios presentes en áreas protegidas de Colombia; caso Municipio de San Juanito, Meta
}

\section{Disturbances present in protected areas of Colombia; case Municipio de San Juanito, Meta}

\author{
CASTRO-GARZÓN, Hernando ${ }^{1}$ \\ MONTEALEGRE-TORRES, Francy L. ${ }^{2}$ \\ RODRÍGUEZ, Juan P. ${ }^{3}$
}

\begin{abstract}
Resumen
En este trabajo se documentó el régimen de disturbios generados en los diferentes predios ubicados en zona de protección del municipio de San Juanito Meta, Colombia; utilizando como base la clasificación establecida por Vargas (2013). Se tomaron como base de estudio las veredas que presentaban mayores intervenciones en cuanto al número de predios presentes y su concentración. Se utilizaron técnicas para la recopilación de la información como la observación, entrevistas y grupos focales.

Palabras clave: áreas protegidas, disturbios, sostenibilidad, comunidad.
\end{abstract}

\begin{abstract}
In this work there researched the disturbances generated in the different properties located in protection area of the municipality of San Juanito Meta, Colombia; using the classification established by Vargas (2013). There took as a study base that were presenting major interventions as for the number of present properties and its concentration. Skills were used for the compilation of the information like the observation, interviews and focal groups.

Key words: protected areas, disturbances, sustainability, community.
\end{abstract}

\section{Introducción}

Al oriente de Colombia se ubica el departamento del Meta considerado la puerta entre la zona de la Orinoquia y la Amazonia (Chisnes, 2018, p.35), con sus respectivas zonas de transición; al norte del mismo en el punto más alto ya que posee territorios en suelos andinos se ubica el municipio de San Juanito (Arboleda, 2018, p.240) que por sus características orográficas así como de biodiversidad se constituye en un territorio estratégico para la producción de agua como de una gran oferta de servicios ecosistémicos, de allí se abastece el $80 \%$ del agua para la ciudad de Bogotá (Moreno, 2017, p.371); en el municipio se presenta un número de 677 predios (Alcaldía San Juanito, 2016); se aclara que los predios presentan desenglobes y tenencias de hecho que crean un número mayor de los mismos (EOT, 2005), en las siguientes veredas: San Luis de Toledo, San Luis El Plan, La Candelaria,

\footnotetext{
${ }^{1}$ Profesor, Escuela de Administración y Negocios. Facultad de Ciencias Económicas, Universidad de los Llanos, Villavicencio, Colombia. Correo electrónico: hcastro@unillanos.edu.co

2 Maestría en Gestión para el desarrollo sustentable, Universidad Autónoma de Guerrero- Acapulco, México f.lilianatorres@gmail.com

3 Profesor Titular. Facultad del Medio Ambiente y Recursos Naturales. Universidad Distrital Francisco José de Caldas. Bogotá, Colombia. Correo electrónico: jprodriguezm@udistrital.edu.co
} 
El Carmen, San Roque, San Juanito (Centro), El Tablón, San José y Los Rubios (Alcaldía San Juanito-Meta, 2017; SIG Unillanos, 2017). Las zonas de protección objeto de estudio son las del Páramo De Chingaza, delimitado en la Resolución 710 de 2016 y el Parque Nacional Natural Chingaza, erigido como Parque Nacional Natural mediante la Resolución 154 de junio de 1977 y el Decreto 622 de 1977 reglamentario de la Ley 281 de 1974 que establece el sistema de Parques Nacionales, estas están insertas en el 50\% del territorio municipal. El número de predios en la zona de estudio es de 58 con una extensión que va desde 1,7 hasta las 4.609 hectáreas (SIG Unillanos, 2017).

\section{Metodología}

Frente a la escogencia de los actores participantes se estableció como estrategia mezclar dos formas de muestreo como son: el intencionado (Patton, 2006, p.1) y el de bola de nieve (Goodman, 1961, p.150), se establecieron tenentes o propietarios de predios ubicados en las zonas de protección del municipio de San Juanito; se visitaron predios ubicados en las veredas San Luis el Plan, San Luis de Toledo, El Carmen y La Candelaria; las visitas realizadas fueron a los predios de mayor acceso, ya que estas zonas presentan pendientes de más del $50 \%$ (James, Niño \& Reinoso, 2013, p.10) y las vías son caminos de herradura.

Por otro lado, la selección se realizó a partir de la identificación realizada por parte de un informante principal que posee un amplio conocimiento del municipio, de la población y sus interacciones con zonas de protección. Revisada la información predial se realizó la respectiva visita de campo para observar la composición de los predios seleccionados, luego se procedió a contactar con los tenentes o propietarios de los predios fungiendo como mediador el informante inicial, quien actuó como guía y contacto con la comunidad procediendo mediante: entrevistas, focus group y observación, con los informantes seleccionados

\section{1. Áreas protegidas}

Colombia con una superficie total de más de un millón ciento cuarenta mil kilómetros cuadrados de los cuales el $8.8 \%$ es agua (Ministerio de Comercio Industria y Turismo, 2018) y el 11,3\% son áreas protegidas, las cuales están administradas y supervisadas por el Sistema Nacional Áreas Protegidas (SINAP) el cual agrupa este porcentaje en un total de 59 áreas protegidas (Vásquez M \& Andrade, 2016, p.130).

El modelo de conservación planteado por los gobiernos es altamente restrictivo en cuanto al uso y manejo de los recursos, proponiendo reglas que establecen quién, cómo, cuándo y dónde puede usarlos (Adams \& Hutton, 2007, p.147), esta función desarrollada en Colombia por el SINAP instaura la conservación como única actividad permitida dentro de las Áreas de Protección (AP) (Parques Nacionales Naturales de Colombia, 2015) coaccionando a los habitantes de estas zonas en el desarrollo de sus actividades económicas, culturales y sociales.

De esta manera se genera un conflicto en la declaración de AP puesto que la interacción de ser humanonaturaleza se ve interrumpida por las políticas de conservación, así lo permite observar Gutiérrez Antolínez, (2016, p.23). El mecanismo de sustraer un territorio como área protegida se hacía hasta el siglo pasado teniendo en cuenta solo criterios científicos y de altura, tales como territorios rurales superior a los $1800 \mathrm{msnm}$ y la conservación de la biodiversidad del lugar, no se valoraba la presencia de habitantes y la afectación a sus actividades productivas. Esta visión cambio en el 2003 durante el -V congreso mundial de Parques Nacionales y Áreas Protegidas- en donde el involucramiento del factor humano comienza a hacer parte de los ejes de planeación y conservación de estas zonas (Jenkins \& Joppa, 2009, p.2167).

Así las cosas, para las administraciones de los gobiernos las AP son objeto de normalización sin afectación a la población, permitiendo desarrollar estrategias de involucramiento de los agentes y actores acercando las políticas de conservación y desarrollo rural. 


\subsection{Tipos de disturbios}

Las zonas altas son regiones severamente afectadas y degradadas por el manejo de cultivos y la explotación ganadera (Marín, et al, 2017, p.6), ya que las prácticas culturales para estos emplazamientos conlleva tala y quema de las plantas endémicas para una posterior fertilización para que sea productiva con especies exóticas; perdiendo las características de permeabilidad y absorción del suelo generando degradación eco sistémica (Humbolt, 2008, p.208) estas acciones antrópicas crean desbalances en la producción de agua que se ve reflejada en las épocas estacionales, invierno y verano, ya que se presentan fenómenos erosivos y remoción en masa o sequías extremas respectivamente(López-Sáez, et al, 2017, p.75); siendo los disturbios (Lizana-Martínez, 2017, p.3) los generadores de cambio en las estructuras principales o en las diferentes componentes de los ecosistemas; estos presentados en un lapso de tiempo o también periódicos, definido en un área o territorio, así como el impacto ejercido sobre las unidades intervenidas. Los disturbios antrópicos se enmarcan en un régimen establecido en (Vargas, 2013, p.55) los cuales son: fuego, ganadería, agricultura, minería, plantaciones exóticas, construcción de obras civiles, deforestación, especies invasoras, cacería; se considera el cambio climático (Salisbury, et al, 2018, p.111) como un macro disturbio que puede alterar la intensidad y la extensión de los anteriores o crear nuevos no conocidos.

Los regímenes de disturbios en tierras altas afectan la producción de lluvia horizontal (Rivera-Ospina, 2017, p.10) que en estos gradientes es la base fundamental para la producción de agua, estos en escenarios de cambio climático (Agudelo, et al, 2016, p.112) son proclives a una mayor pérdida de su estructura principal intensificando la perdida de la red hídrica de la zona productora.

\section{Resultados}

En las observaciones realizadas se encontró un común denominador en la tipología de su explotación; se determinó que no existen viviendas permanentes en ellos, se presenta una especie de bodegas de un área muy reducida, que solamente podrían alojar a una persona; así mismo se encontraron cultivos de pastos con extensiones moderadas de áreas plantadas; otro rasgo encontrado es el de la ganadería extensiva en baja densidad, en algunos casos ganado ovino en los predios más cercanos. Por otra parte, se apreciaban los restos de árboles en los potreros o tocones de éstos (García \& Méndez, 2015, p.132) y algunos presentaban muestras de quema y otros de ser solamente talados, y en algunos casos al lado de los potreros no se presenta cobertura vegetal. Determinando que según lo presentado por (Vargas, 2013, p.55) el régimen de disturbios observado es: fuego, ganadería, deforestación y especies invasoras.

En cuanto a los informantes contactados se estableció por su parte que: "las ganaderías no sobrepasan las 20 cabezas por predio y algunos poseían ovejas pero que en la mayoría son reses"; no se presenta agricultura porque "a esa altura no se produce nada sólo sirve para cría de ganado"; además añadieron que "las temperaturas han aumentado en temporada de sequía, consecuencia del cambio climático". Adicionaron en cuanto a la quema y deforestación: "lo que hoy es zona de protección antes se quemaba o talaba. La costumbre de todos era quemar, no importaba si era zona protegida o no. Sin embargo, hoy hay mayores controles en ese tema". Agregando "que se hacía para establecer potreros de pasto kikuyo que se adapta muy bien a estas zonas". Y se reiteró en cuanto a cacería que: "San Juanito fue fundado a raíz de la cacería, esta es una práctica que se hace por deporte y no por lucro"; todos manifestaron cazar o haber cazado por gusto, dicen que: "antes había muchos animales como lapas, gallitos de roca, pero ahora no se ven tanto". Acorde con la información presentada por los informantes se configuran adicionalmente disturbios tipo "cambio climático y cacería" (Vargas, 2013, p.56); un informante determinó que siembra papa, pero no se observó en ninguno de los predios visitados. Por lo anterior se concluye que hay cohesión entre lo observado y lo referenciado por los tenentes o propietarios de predios en zonas de protección. 
Figura 1

Mapa predios en Áreas de Protección

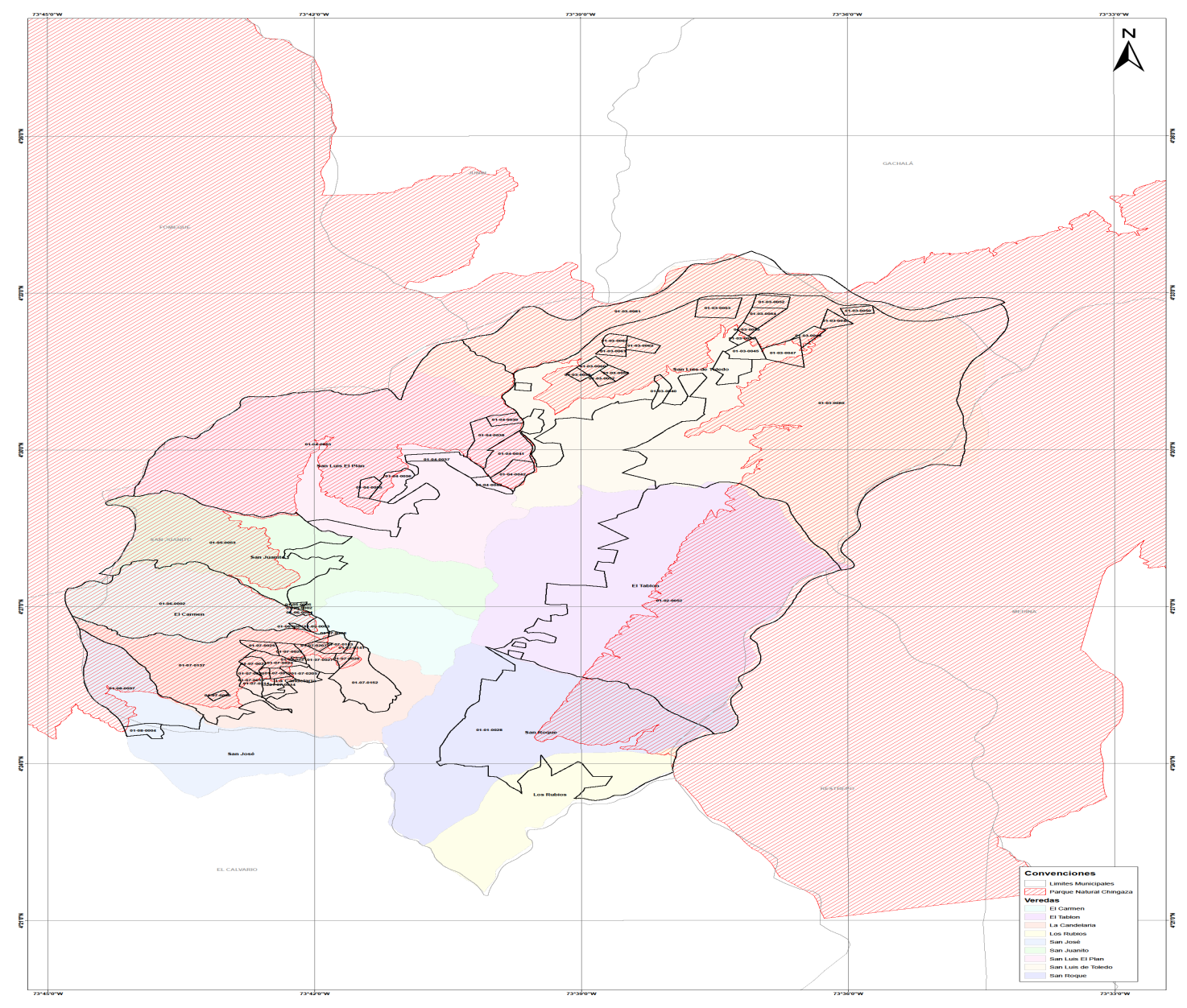

Fuente: UNILLANOS 2017

A continuación se presenta gráficamente los regímenes de disturbios contrastados teóricamente, mediante la observación y las "expresiones" (Belzunegui, Brunet \& Pastor, 2012, p.22) brindadas por los actores participantes en la investigación en cuatro (4) veredas y la opinión de residentes de la zona urbana de San Juanito, ya que se consideró importante esta visión. 
Figura 2

Vereda El Carmen

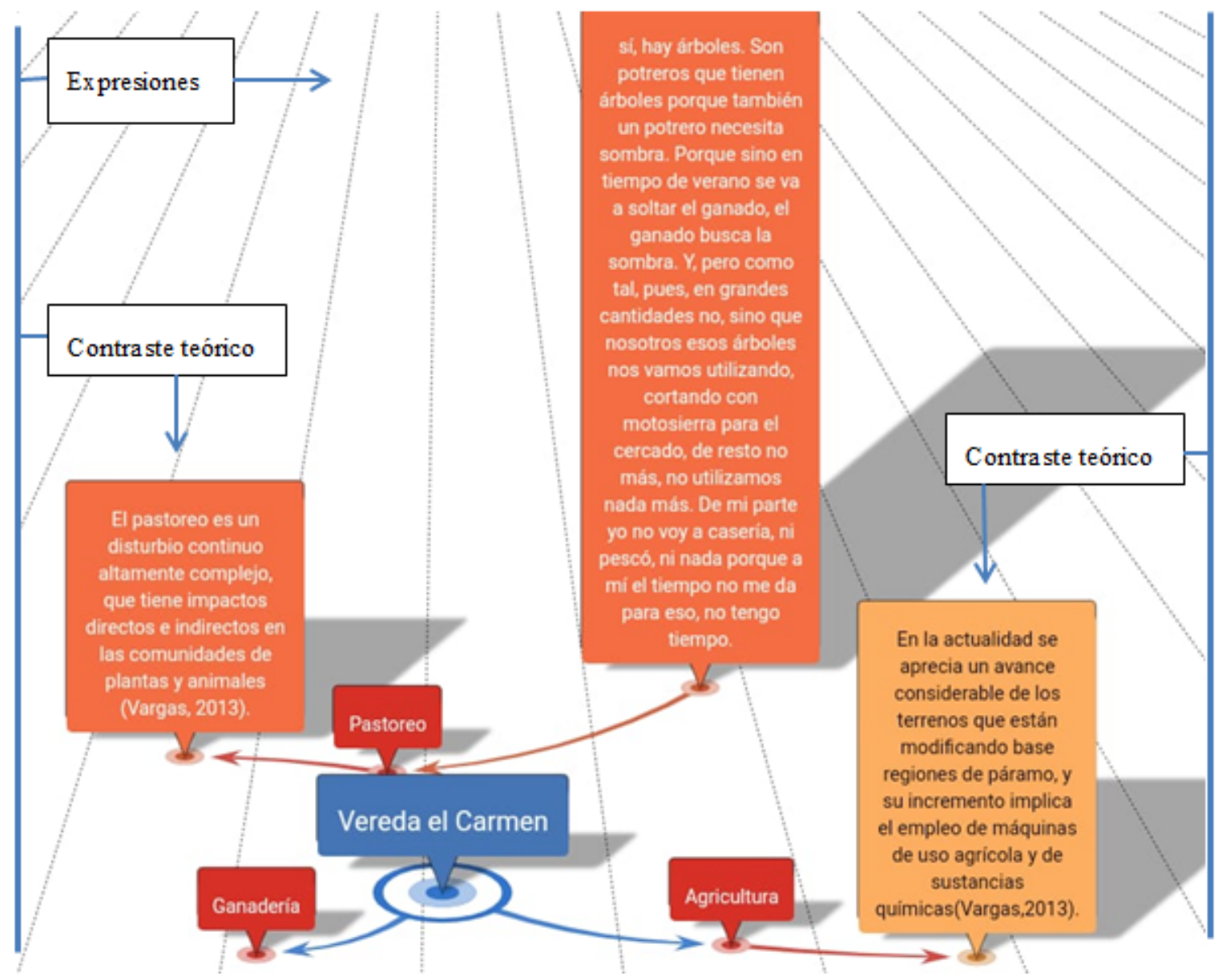

Fuente: Elaborado por los autores 
Figura 3

Vereda La Candelaria

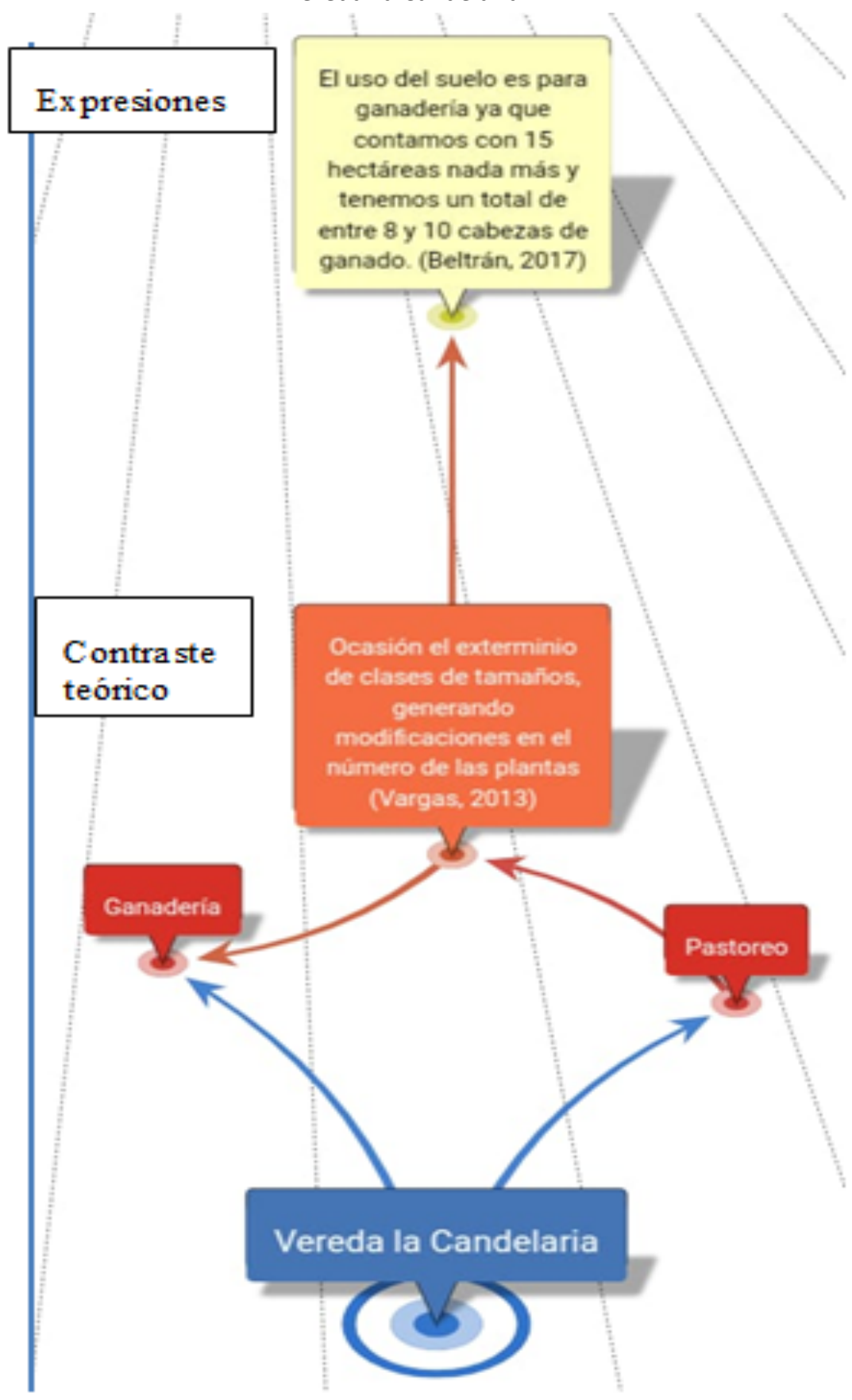

Fuente: Elaborado por los autores 
Figura 4

Zona urbana de San Juanito

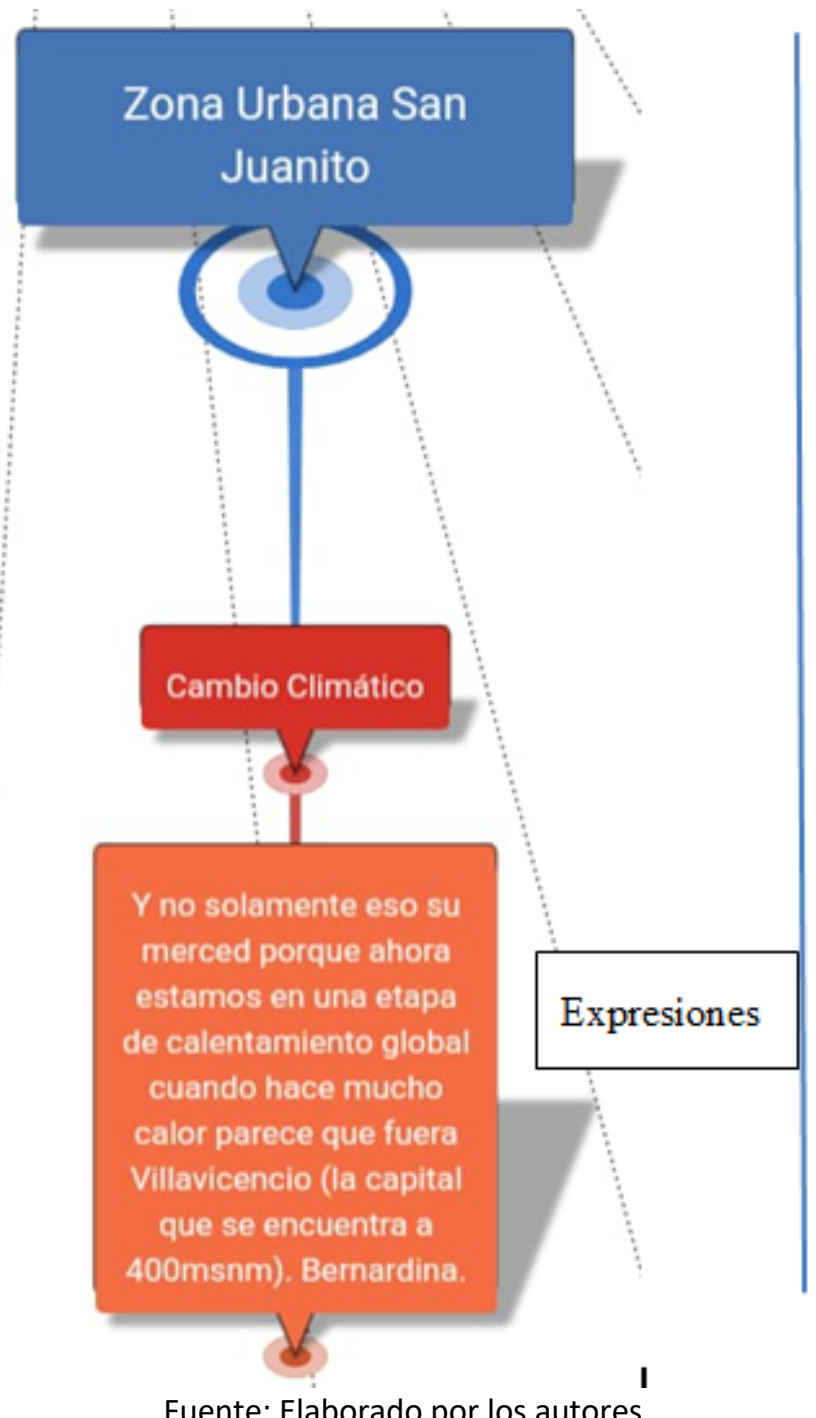

Fuente: Elaborado por los autores 
Figura 5

Vereda San Luis el Plan y San Luis de Toledo

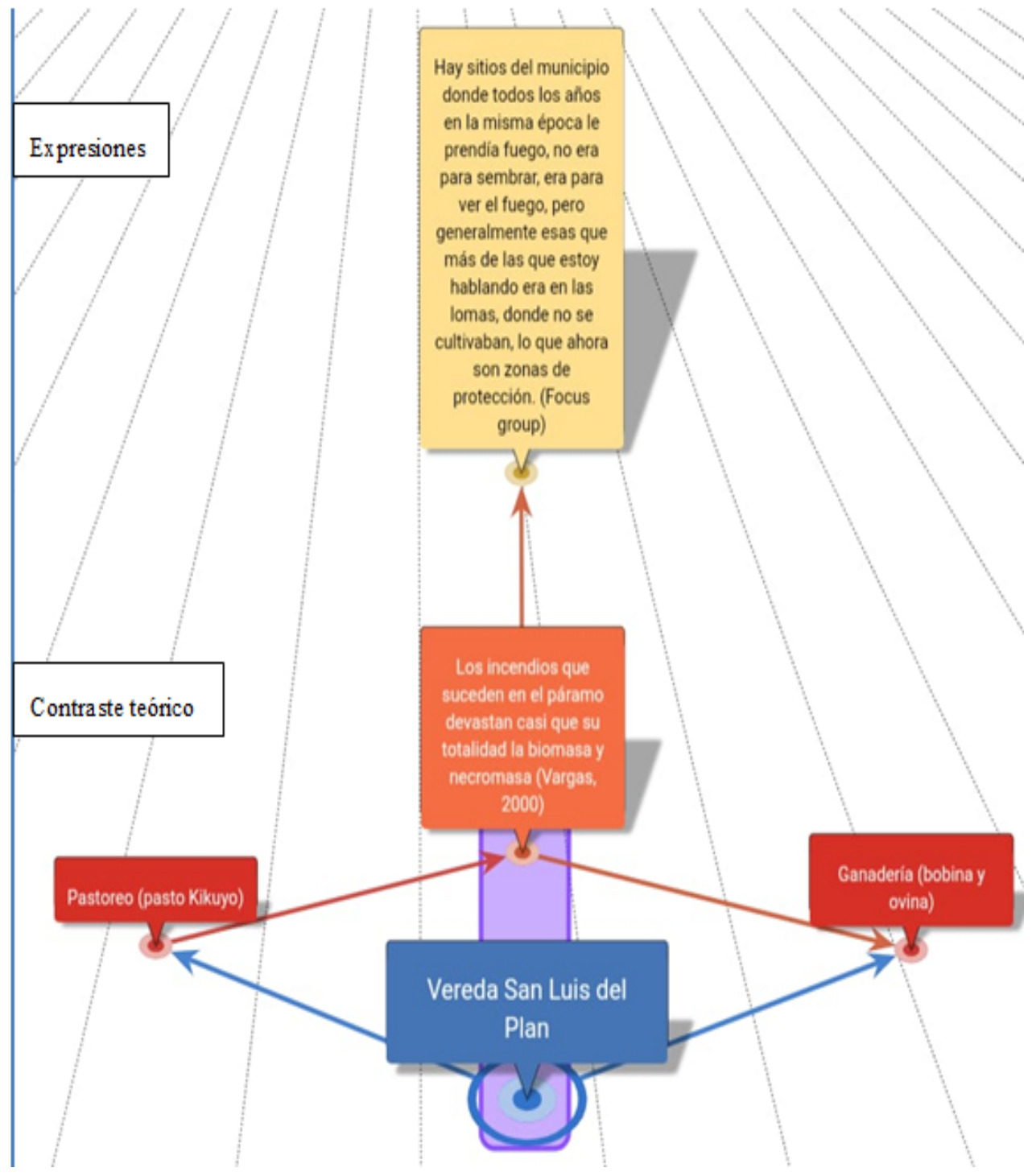

\section{Conclusiones}

Se puso de manifiesto por parte de la comunidad el deseo de enajenación de los predios incluidos en Áreas de Protección, pero desconocen los condicionamientos de legalidad y asumen que la posesión de tierras es suficiente para la negociación. Se estableció que la comunidad no posee saberes tradicionales sobre las formas de explotación del territorio; han sido sucesiones de ensayo y error cada vez que cambian la vocación productiva; por ende, la proclividad a los impactos negativos.

Para el establecimiento de Áreas de Protección es necesario tomar en cuenta las apropiaciones históricas de los territorios y su desarrollo, en cuanto a relaciones de producción. Son necesarias políticas de implementación de procesos de aprendizaje consensuado e incluyente de las comunidades insertas o conexas a las zonas excluidas. Lo anterior con el fin de crear procesos en caso de que no existan de sostenibilidad, procurando generar equilibrios dinámicos entre la preservación y el uso de los suelos según la ubicación de los sistemas socio ecológicos. 
El concepto de intervención debe ser entendido desde la óptica de la ejecución de política de Estado; no se pueden desconocer los procesos de interacciones entre comunidades y territorio desde centurias.

\section{Referencias bibliográficas}

Adams, W. M., \& Hutton, J. (2007). People, parks and poverty: political ecology and biodiversity conservation. Conservation and society, vol. 5, no 2, p. 147.

Agudelo, N., Mora, J. M., Pérard, S., \& Solórzano, J. C. J. (2016). Extensión del Bosque Nublado y su Contribución de la Lluvia Horizontal a la Precipitación Total en la Reserva Biológica Uyuca, Honduras. Ceiba, vol. 53, no 2, p. 109-123.

Alcaldía de San Juanito. (2005). Informe poblacional base. Página Oficial del Municipio, 2016. http://www.sanjuanito-meta.gov.co/index.shtml

Alcaldía San Juanito. (2017). Informe de espacios por veredas. http://www.sanjuanito-meta.gov.co/

Arboleda, D; Ortiz, Á. (2018) Convergencia regional en el Departamento del Meta, Colombia: un enfoque desde el desarrollo humano. Lecturas de Economía, no 89, p. 221-254.

Belzunegui, E; Brunet, I; Pastor, I. (2012). El diseño del Análisis Cualitativo Multinivel: una aplicación práctica para el análisis de entrevistas. EMPIRIA. Revista de Metodología de las Ciencias Sociales, no 24, p. 15-44.

Chisnes, L. (2018). Apertura económica y cultivos en el departamento del Meta-Colombia, 1990-2000. Historia y espacio, 14 (50), pp. 32-40.

EOT- Esquema de Ordenamiento Territorial. (2005). EOT del municipio de San Juanito. Alcaldía Municipal.

García, A; Méndez, J. (2015). Estructura diamétrica de árboles en potreros de la región Bajo Mixe, Oaxaca. Teoría y Praxis, no 18, p. 131-151.

Goodman, L. (1961) Snowball sampling. Annals of Mathematical Statistics. 32(1), 148- 170.

Gutiérrez-Antolínez, C. (2016). Conflictos socioambientales derivados de la declaración del Parque Nacional Natural Chingaza en zonas de producción campesina.Universidad Nacional de Colombia Facultad de Ciencias Económicas.

Humboldt- Instituto De Investigación De Recursos Biológicos Alexander Von Humboldt. (2008). Atlas de Paramos de Colombia, pp. 208.

James, M; Niño, M; Reinoso, E. (2013). Una aproximación para la obtención de mapas de desplazamiento traslacional de laderas a nivel regional inducido por sismos. Ingeniería sísmica, no 89, p. 01-24.

Jenkins, C. N., \& Joppa, L. (2009). Expansion of the global terrestrial protected area system. Biological Conservation, 142(10), 2166-2174

Lizana Martínez, C. A. (2017). Propuesta de zonas prioritarias para la recuperación de bosque nativo degradado en la Región de Los Ríos, Chile.

López-Sáez, J. A., Abel-Schaad, D., Iriarte, E., Alba-Sánchez, F., Pérez-Díaz, S., Guerra-Doce, E., \& Abarquero Moras, F. J. (2017). Una perspectiva paleoambiental de la explotación de la sal en las Lagunas de Villafáfila (Tierra de Campos, Zamora). Cuaternario y Geomorfología, vol. 31, no 1-2, p. 73-104.

Marín, M. A. M. (2017). Pescador, L. R., Ramos, L. R., \& Charry, J. L. A. Impacto de la actividad ganadera sobre el suelo en Colombia. Ingeniería y Región, vol. 17, p. 1-12 
Ministerio de Comercio industria y turismo.(2018). Plan Nacional Sectorial del Turismo 2018 -2022, Bogotá. Retrieved from https://www.academia.edu/37607682/Plan_Nacional_Sectorial_del_Turismo_2018_-2022

Moreno, H. (2017) Implicaciones de las políticas de conservación ambiental en la relación de los habitantes de Guasca con su entorno. AlBR. Revista de Antropología Iberoamericana, vol. 12, no 3, p. 365-387.

Parques Nacionales Naturales de Colombia. (2015). Áreas Protegidas: Territorios para la Vida y la Paz. Tomo I: Áreas protegidas para el desarrollo. Retrieved from http://www.parquesnacionales.gov.co/portal/wpcontent/uploads/2015/11/PNNC_TOMO1_VF2.pdf

Patton, M. (2006). Prefacio: Tendencias y problemas como contexto. Investigación en las escuelas, vol. 13, no 1, p. 1-2.

Rivera-Ospina, D. (2017). Definición de criterios para la delimitación de los diferentes tipos de Páramos del País y de lineamientos para su conservación: parte 1 Propuesta de tipología socioecológica de los Páramos del País. Propuesta de criterios que deben guiar los procesos de delimitación del Páramo. Análisis de limitaciones y oportunidades de aplicación de criterios de delimitación de Páramos.

Salisbury, DS, Aguirre, CN, Gudiño, ME, Martín, FL, Pyszczek, OL, Sanabria, R., y Snaider, PP. (2018). El Atlas del Cambio Climatico de las Americas. Revista Geográfica, no 159, p. 109-126.

SIG Unillanos- Sistema de Información Geográfica de la Universidad de los Llanos. (2017) Veredas del municipio de San Juanito, Meta.

Vargas, O. (2013) Disturbios en los páramos andinos. En Cortés-Duque, J. \& Sarmiento, C. (eds). Visión Socioecosistémica de los Páramos y la Alta Montaña Colombiana: Memorias del Proceso de Definición de Criterios para la Delimitación de Páramos. Instituto de Investigación de Recursos Biológicos "Alexander von Humboldt. Bogotá D.C. Colombia, 55 p.

Vásquez M, J. L., \& Andrade, G. I.(2016). El paisaje protegido, pieza faltante en el Sistema de Áreas Protegidas de Colombia Resumen. Biodiversidad En La Práctica Documentos de Trabajo Del Instituto Humboldt, 1(2015), pp,128-146. 